\title{
Zur Osteosynthese bei Frakturen im zahnlosen atrophen Unterkiefer - Möglichkeiten zum Hardware Downsizing, Lingual Plating
}

\author{
Carl-Peter Cornelius, Florian Andreas Probst, Jean Paul Christian Rathgeber, \\ Marc Metzger
}

\section{Zusammenfassung}

Die Frakturbehandlung im zahnlosen atrophen Unterkiefer ist problematisch. Dies hat seine Gründe in lokalen Faktoren, wie der reduzierten ossären Quantität und Qualität sowie in altersassoziierten Komorbiditäten. Die Therapiemöglichkeiten reichen von minimalistisch konservativen Ansätzen über die Applikation von Miniplatten unter Verwendung transoraler chirurgischer Zugänge bis hin zur aggressiven transfazial-submandibulären Dissektion mit Reposition und Montage von Makro- bzw. Rekonstruktionsplatten. Wenn Rekonstruktionsplattensysteme mit einem hochatrophen „Pencilbone“-Unterkiefer zusammentreffen, wird ein „Overengineering“ deutlich, da die Massivität des Osteosynthesematerials und der residuale Knochen in krassem Missverhältnis zueinander stehen. Wegen der hohen Stabilität der mit Rekonstruktionsplatten überbrückten Frakturen gelten diese jedoch nach wie vor als Referenz für Vergleiche mit anderen internen Fixationsverfahren bzw. Plattenvarianten und Konstrukten.

\section{On the Osteosynthesis of Atrophic Mandible Fractures - Options for Hardware Downsizing, Lingual Plating}

The management of atrophic mandible fractures is challenging. This is attributed to local factors like impaired bone quality and quantity as well as to age-related comorbidities. Therapeutic options range from minimalistic non-operative approaches over the transoral application of miniplates to extended submandibular approaches with application of reconstruction plates. Stabilising a "pencil-bone" mandible with a large macroplate may appear like "over-engineering" due to the size discrepancy between bone and plate. However, treatment of atrophic mandible fractures with reconstruction plates can still be considered as the standard because of the high stability that is achievable.

\section{Spezielle Aspekte bei Osteosynthesen im zahnlosen atrophen Unterkiefer}

Frakturen im zahnlosen atrophen Unterkiefer machen nur einen geringen prozentualen Anteil am Gesamtaufkommen aller Gesichtsschädelfrakturen aus (ca. $1 \%$ [1] und ca. 3\% [2]).

Prädilektionsgebiet für Frakturen im zahnlosen mäßig atrophen Unterkiefer ist die Korpusregion dorsal des Foramen

OP-JOURNAL 2013; 29: 164-174

(c) Georg Thieme Verlag KG Stuttgart · New York DOI http://dx.doi.org/10.1055/s-0033-1350819 mentale [3]. Mit fortschreitenden Atrophiegraden wandern die Frakturen offenbar weiter nach anterior und durchqueren direkt die foraminale Region oder die nervale Austrittsöffnung [4]. Die genannten Unterkieferabschnitte sind im zahnlosen Unterkiefer mit 62\% [4] bis $86 \%$ [5] weitaus häufiger von Frakturen betroffen als in der bezahnten Mandibula.

Die Mehrheit der Patienten mit Frakturen im zahnlosen atrophen Unterkiefer gehört zur älteren Bevölkerungsgruppe (> 65-70 Jahre), weshalb mit einer hohen Inzidenz von systemischen Komorbiditäten zu rechnen ist, z. B. kardiovas- kulären oder chronisch respiratorischen Erkrankungen und Diabetes mellitus.

Oftmals liegt zusätzlich eine Fehl- oder sogar eine Unterernährungssituation vor $[5,6]$.

Überdies können ungünstige lokale Faktoren, wie die Abnahme der vertikalen Knochenhöhe, eine veränderte Knochenqualität (altersassoziierte Osteoporose, aber auch Sklerosierung bzw. Dichtezunahme der Knochen-Kortices, [7]), Abnahme der Vaskularisation $[8,9]$ oder eine progene intermaxilläre Relation des Unterkiefers zur Oberkieferposition [10] zu einem erhöhten Risiko für Komplikationen bei einer operativen Frakturversorgung beitragen.

\section{Atrophiegrade - Einteilung nach Luhr $[11,12]$}

Die Reparation und das Remodelling (extern und intern) des residualen Unterkiefer-Alveolarfortsatzes nach Verlust oder Extraktion aller Zähne verläuft nach stereotypen Gesetzmäßigkeiten mit periostal-osteoklastischer Resorption, die in der horizontalen Dimension beginnt und sich später vertikal fortsetzt. Die horizontale Resorption soll durch muskulären Druck von Lippen, Wange und Zunge zustande kommen, der Resorptionsvorgang in Vertikalrichtung wird als Inaktivitätsatrophie gedeutet. Die Resorptionsraten sind in den ersten Monaten nach Zahnverlust am größten, verlangsamen sich nach 6 Monaten deutlich und erreichen eine Stabilitätsphase nach 1-2 Jahren [13]. Die progredienten Resorptionsstadien im Unterkiefer zeigen charakteristische Formen in den Knochenquerschnitten, für die mehrere Klassifikationen vorgeschlagen wurden $[14,15]$. Am bekanntesten ist die Einteilung von [16], nach der 6 Resorptionsklassen unterschieden werden. Diese Einteilung geht aus von Anfangsphasen mit gerundetem Kieferkamm über „messerscharfe Kammformen“ mit 


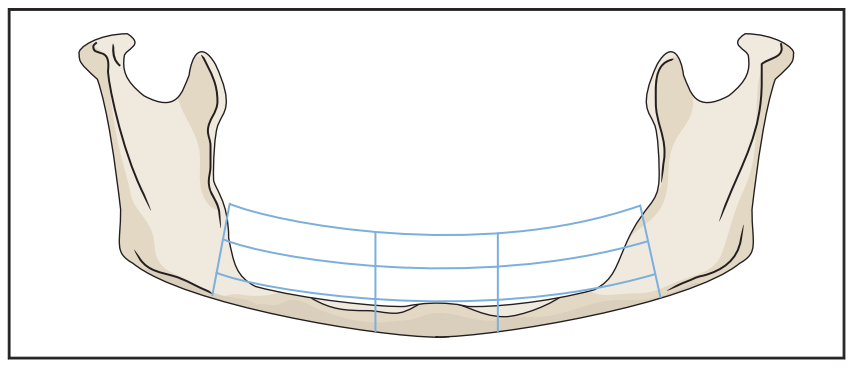

Abb. 1 Atrophieklassen bei Frakturen im zahnlosen Unterkiefer nach Luhr et al. (1996).

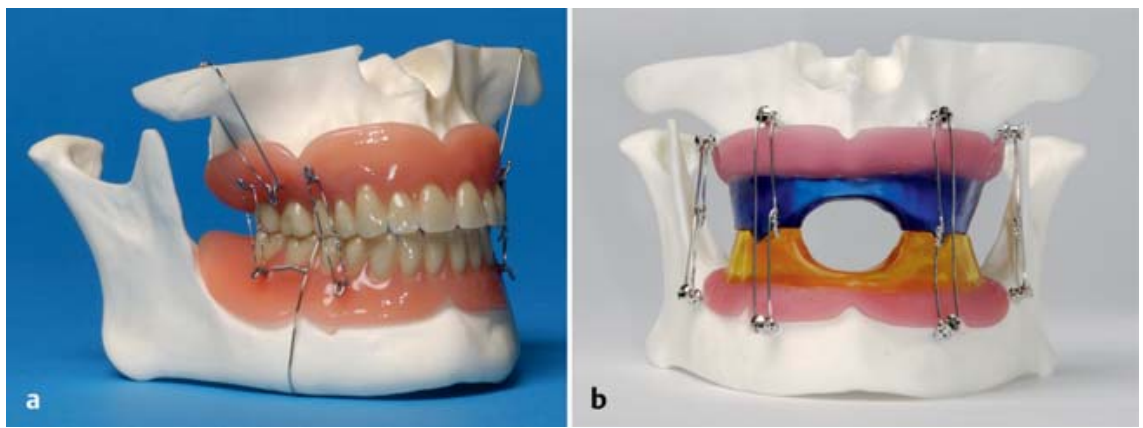

Abb. $\mathbf{2}$ a und $\mathbf{b}$ Möglichkeiten zur mandibulomaxillären Fixation im zahnlosen atrophen Unterkiefer. a Modifikation vorhandener Totalprothesen durch Einpolymerisation von Häkchen, kraniofaziale Fixation über zygomatikomaxilläre Aufhängungen und Drahtumschlingung des Unterkiefers (circummandibular wiring) sowie Drahtcerclagen zur mandibulomaxillären Fixation (MMF) zwischen den Häkchen an den Prothesen; b Gunning-Splint mit Futterloch, in die Alveolarfortsätze ober- bzw. unterhalb der Kunststoffsättel inserierte MMF-Schrauben und Drahtcerclagen über diese Ankerschrauben.

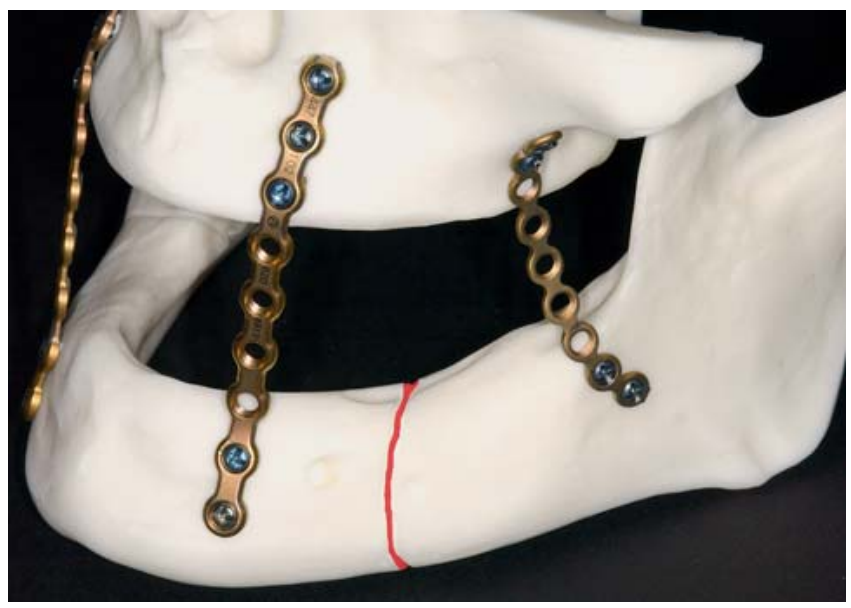

Abb. 3 Applikation von Mini-(Locking-) platten zur Distanzüberbrückung zwischen Ober- und Unterkiefer unter Aufrechterhaltung der vertikalen Kieferrelation als Maßnahme zur Fragmentimmobilisation durch maxillomandibuläre Fixation im zahnlosen Kiefer.

erhaltener Höhe und reduzierter Breite bis hin zur vollständigen Abflachung der alveolären Knochenpartien, um bei hochatrophen Extremvarianten mit Resorption bis in die basalen Unterkieferstrukturen, „negativem“ Höhenprofil und direkt unter dem Mukoperiost frei liegenden $\mathrm{N}$. alveolaris inferior zu enden.

In der Traumatologie wird der Atrophiegrad des zahnlosen Unterkiefers üblicherweise gemäß der Einteilung von Luhr [11,12] angegeben (Abb. 1, Tab. 1).
In Abhängigkeit der variierenden Atrophiegrade und infolge der möglichen Risikokonstellationen durch systemische und weitere lokale Faktoren gilt die adäquate Frakturbehandlung im zahnlosen Unterkiefer als besondere Herausforderung und gibt seit vielen Jahren Anlass zu Kontroversen [17].

\section{Therapeutische Optionen}

Grundsätzlich sind die konservativen, geschlossenen Methoden von den operativen Techniken mit offener Reposition
Tab. 1 Atrophiegrade bei Frakturen im zahnlosen Unterkiefer nach Luhr et al. [11, $12]$.

\begin{tabular}{ll}
\hline Atrophiegrad & $\begin{array}{l}\text { Vertikalhöhe in } \\
\text { der Frakturzone }\end{array}$ \\
\hline $\begin{array}{l}\text { keine traumatologisch } \\
\text { relevante Atrophie }\end{array}$ & $>20 \mathrm{~mm}$ \\
\hline Klasse I & $16-20 \mathrm{~mm}$ \\
\hline Klasse II & $11-15 \mathrm{~mm}$ \\
\hline Klasse III & $\leq 10 \mathrm{~mm}$
\end{tabular}

und Fixation zu unterscheiden. Bei den konservativen Techniken reicht das Spektrum von der Verordnung weicher Kost [18] über das Tragen von KopfKinn-Kappen bis zum „Eingliedern“ von vorhandenem Zahnersatz wie Unterkiefer-Totalprothesen oder von besonders angefertigten Gunning Splints (Gunning 1863) zur mandibulomaxillären Fixation (Abb. 2).

Zur Befestigung von Prothesen bzw. Gunning Splints verwendete man lange Zeit Drahtumschlingungen des Unterkiefers (circummandibular wiring). Diese wurden perkutan mithilfe von Führungsahlen eingebracht. Seit Mitte der 1980er-Jahre werden Osteosyntheseschrauben verwendet. Bei einer geschlossenen Behandlung werden die dislozierten Fragmentenden manuell oder durch Instrumentenzug (z.B. mittels Klemmen) in Kontakt gebracht, bevor die Frakturzone ruhig gestellt wird. Ein einfaches und interessantes Verfahren zur Fragmentimmobilisation durch maxillomandibuläre Fixation im zahnlosen Kiefer ist die Applikation von Miniplatten zur Distanzüberbrückung zwischen Ober- und Unterkiefer unter Aufrechterhaltung der vertikalen Kieferrelation $[19,20]$. Dazu werden die Plattenenden von ca. 4-5 cm langen Miniplatten an gegenüberliegenden Stellen auf der vestibulären Schleimhautseite der Alveolarfortsätze des Ober- und Unterkiefers bis hoch in die Umschlagfalten ankonturiert und mit mindestens 2 Schrauben auf jeder Seite transmukös - vorzugsweise mit Locking-Schrauben, damit die Platte im Abstand von der Schleimhaut gehalten wird - befestigt (Abb. 3).

Historisch war der Fixateur externe eine der ersten Möglichkeiten zur operativen Behandlung von Unterkieferfrakturen ([21,22] u.v.m.) und kam insbesondere dann zur Anwendung, wenn Frakturen vorlagen, die mit den damals gebräuchli- 
Tab. 2 Einige Plattensysteme zur Frakturbehandlung im zahnlosen atrophen Unterkiefer (modifiziert nach Madsen et al. 2009). Kompressionsplatten sind nicht allgemein akzeptiert, weisen aber nach den frühen Arbeiten von Luhr [11,12] sehr gute Erfolgsquoten auf. Die Anfangsversion der Pencilbone-Platte war als Kompressionsplatte konfiguriert. Das Design wurde dann abgeändert in eine winkelstabile Trilock-Variante.

Plattensysteme zur offenen Reposition und Fixation

Miniplatten (Champy-Prinzip)

intermediäre Platten (z. B. Synthes

2.0 UniLOCK Medium Profile, Large Profile)

Makroplatten

Rekonstruktionsplatten (>2,4 mm)

- Non-Locking

- Locking

[Kompressionsplatten]

Pencilbone-Platten - Modus Trilock

chen intraoralen Schienen- oder Prothesenverbänden nur unzureichend $\mathrm{zu}$ behandeln waren. Hierzu zählen bspw. dislozierte Brüche im zahnlosen Unterkiefer oder Trümmer- und Defektsituationen. Aktuelle Fixateur-externe-Systeme bestehen aus die Haut penetrierenden Fixationselementen (Metall Pins bzw. Schanz-Schrauben) und einem aufsteckund verschraubbaren äußeren Gerüst in Form eines Metallbogens. Die Systeme zeichnen sich durch eine einfache, schnelle und atraumatische Handhabung ohne Notwendigkeit zu einer größeren Exposition der Knochenoberflächen aus. Wegen der sperrigen, den Patienten beeinträchtigenden äußeren Rahmenkonstruktion beschränkt sich der Einsatz eines Fixateur externe heutzutage freilich auf wenige andere Indikationen ([23-25], Probst et al. 2013 - dieses OP-Journal).

Die offene Reposition von Frakturen im zahnlosen atrophen Unterkiefer bietet zahlreiche Optionen zur internen Fixierung mit Osteosyntheseplatten in unterschiedlichem Design und variierender Dimensionierung (d.h. Mini- und Makroplatten) sowie zur Kombination mit mehreren Typen von Knochentransplantaten (Tab. 2).

\section{Fixationstechniken}

Nach operativer Freilegung und Reposition der Fragmente stehen verschiedene Fixationstechniken mit Osteosynthese-

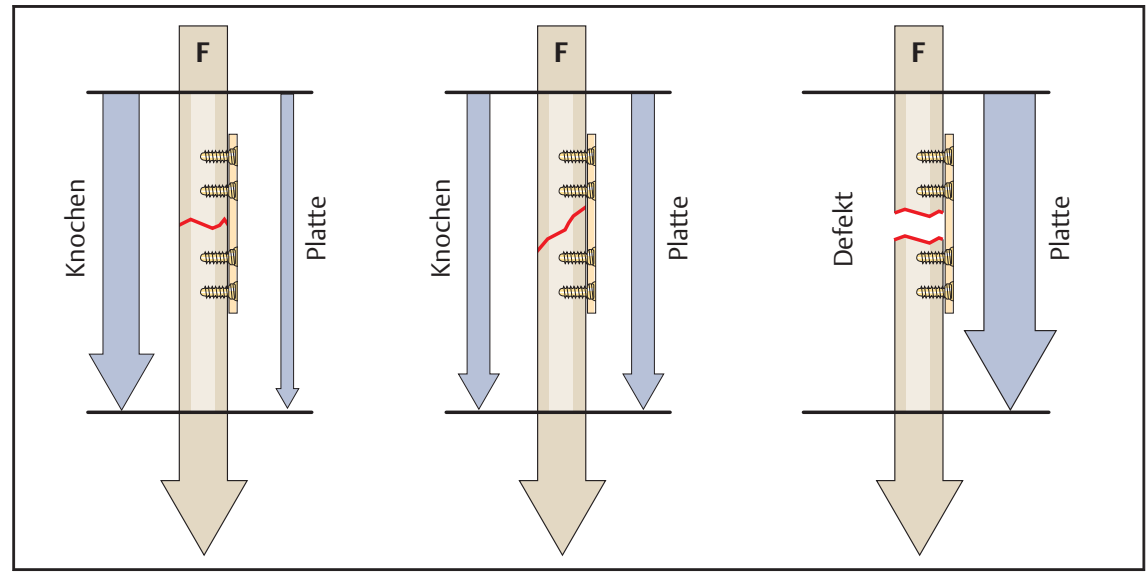

Abb. 4 Prinzip der Kraftübertragung. Links: Load-sharing-Situation; Mitte: intermediäres Szenario - Knochen und Platte tragen zu gleichen Teilen zur Lastübertragung bei. Dementsprechend ist eine größer dimensionierte Platte erforderlich; Rechts: Load-bearing-Situation - weitere Erläuterungen im Text.

platten zur Verfügung (Tab. 2). Entsprechend ihrem Querschnitt bzw. ihrer Profilstärke werden die Osteosyntheseplatten danach eingeteilt, ob sich eine Stabilität bzw. Kraftübertragung im Sinne eines Load Sharing oder Load Bearing [26] erreichen lässt (Abb.4).

Beim Load Sharing übernimmt der Knochen nach adaptiver Verplattung die funktionelle Hauptlast während der Kraftübertragung, beim Load Bearing wird die Kraftübertragung zur alleinigen Aufgabe der Platte. Die Profilstärke der Platten wird im Allgemeinen nicht direkt, sondern über die zur Fixation verwendeten Schraubendurchmesser (in $\mathrm{mm}$ ) angegeben. Die Grenze zwischen Mini- und Makroplatten, die im Schrifttum nicht genau definiert ist, beginnt sicherlich bei einem Schraubendurchmesser oberhalb von $2 \mathrm{~mm}$.

$\mathrm{Zu}$ einer Load-Sharing-Osteosynthese bei Unterkieferfrakturen kommen demnach Miniplatten (Schraubendurchmesser $1.5,1.7,2 \mathrm{~mm}$ ), die nach den Prinzipien von Michelet et al. [27] und Champy et al. [28-32] angebracht werden, zur Anwendung. Die Dimension der Knochenquerschnitte an den Frakturenden muss dabei zur funktionellen Lastübertragung während der Mastikation ausreichen, denn die Osteosynthese mit miniaturisierten Platten kompensiert vornehmlich Zugkräfte und liefert insofern nur einen geringen Beitrag zur Stabilität. Im Gegensatz zur Lastteilung liegen in einer Load-Bearing-Situation im Frakturbereich soweit reduzierte Knochenverhältnisse vor, dass dies einem Defekt gleichkommt und eine nunmehr massiv konfigurierte (Makro-) Osteosyntheseplatte als alleiniger Lastüberträger fungiert. $\mathrm{Zu}$ einer Load-Bearing-Osteosynthese werden üblicherweise Rekonstruktionsplatten (Schraubendurchmesser: $2.3,2.4,2.5,2.7-3 \mathrm{~mm}$ ) verwendet.

Vorteile der offenen Reposition und Fixation von Frakturen im zahnlosen Unterkiefer mit Miniplatten sind die minimale Deperiostierung an der krestalen Knochenoberfläche sowie die technisch einfache und schnelle Durchführbarkeit [2, 33-35]. Bei fortgeschrittener Atrophie des Unterkiefers wird empfohlen, die Miniplatten weiter in Richtung Unterkieferunterrand zu platzieren. Bei reduzierter Knochenqualität sollte statt einer 4Loch-Platte eine 6-Loch-Platte mit einer Fixierung durch 3 Schrauben auf jeder Fragmentseite verwendet werden [36].

Rekonstruktionsplatten erlauben lasttragende Osteosynthesen mit hoher Rigidität und Primärstabilität bei Frakturen im zahnlosen atrophen Unterkiefer (z. B. [4, 37-43]). Mit ausreichend großer Spannweite der Platten kann der Frakturbereich überbrückt werden und die Befestigung entfernt davon in stabilen Knochenarealen erfolgen.

Bei unilateralen Frakturen im atrophen Unterkieferkorpusbereich erfolgt die Schraubenverankerung im R. ascendens und im Kinn- bzw. Symphysenbereich, bei bilateralen Korpusfrakturen wird die Platte von Kieferwinkel zu Kieferwinkel in Form eines Hufeisens appliziert und an den Rami verschraubt. 

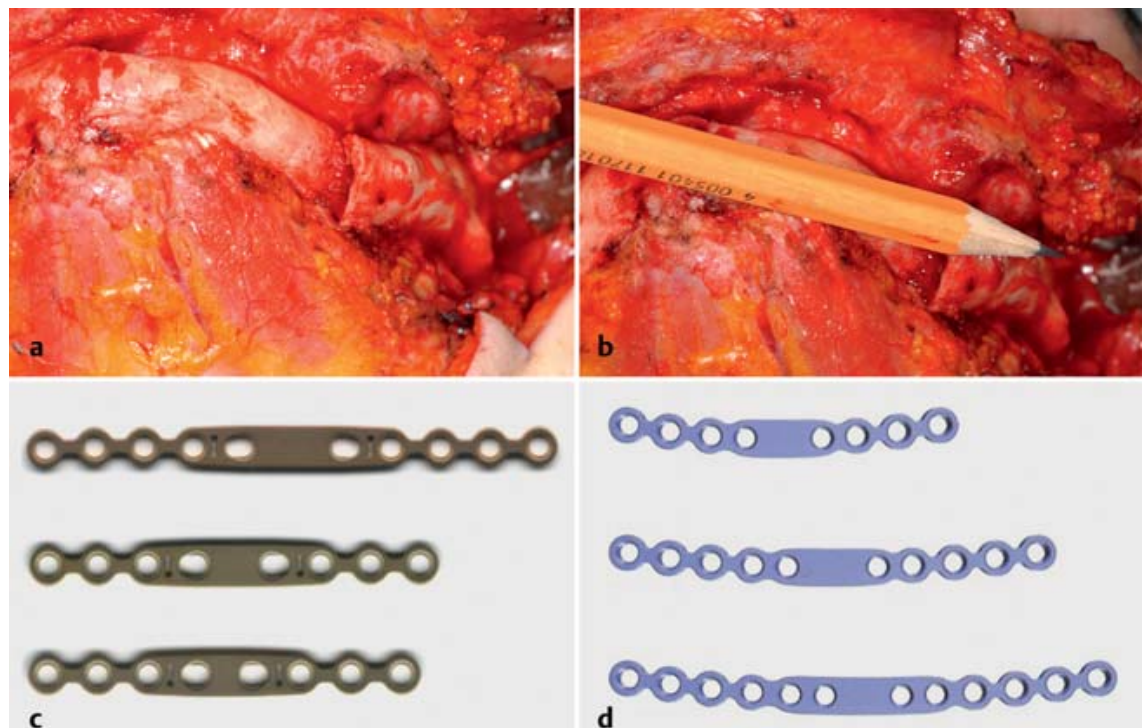

0000000
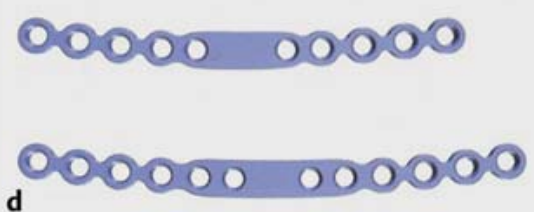

Abb. $\mathbf{5}$ a bis $\mathbf{d}$ Pencilbone und Pencilbone-Platte. $\mathbf{a}$ und $\mathbf{b}$ Fraktur im Korpus eines hochatrophen Pencilbone-Unterkiefers, Bleistift zum Größenvergleich; c Medartis Modus ${ }^{\circledR}$ Trauma 2.0 Pencilbone-Minikompressionsplattensystem: 3 Plattenvarianten mit sphärischen Gleitlöchern im Mittelsteg. Unterschiede in: Breite des Steges zwischen den Gleitlöchern, Länge des verstärkten Mittelstegs, Gesamtlänge der Platten und Anzahl der Löcher im Adaptationsplattenteil; d Neues Pencilbone Plate Design mit Locking-Löchern im Mittelsteg zur winkelstabilen Verblockung mit Trilock-Schraubenköpfen.

\section{Locking Plates}

Seit mehr als 2 Jahrzehnten gibt es Locking-Platten/Locking-Schrauben-Systeme, die bei der Versorgung von Frakturen im atrophen Unterkiefer als „Fixateur interne" fungieren können und biomechanische Vorteile gegenüber konventionellen (Non-Locking) Plattensystemen mit sich bringen. Aus der Locking-Funktion ergibt sich angeblich ein größerer Spielraum bei der Adaptation und Konturierung der Platten an den Knochen. Distanzen bis zu $4 \mathrm{~mm}$ zwischen Platte und Knochenoberfläche sollen tolerierbar sein [44]. Dieser Abstand kann der Vaskularisierung der kortikalen Knochenschicht durch die umgebenden Weichgewebestrukturen zugute kommen [45]. Als weiterer Vorteil kann gelten, dass die Stabilität des Schrauben-PlattenKnochen-Verbunds insgesamt größer ist als bei Standardplatten [46-50], womit bei limitierten anatomischen Platzverhältnissen infolge von Atrophie grundsätzlich auch im Querschnitt reduzierte Plattenprofile möglich werden [42]. Ferner werden sekundäre Dislokationen der reponierten Fragmente durch die Schraubeninsertion vermieden, weil die Fragmente nicht an die Platte herangezogen werden, sobald die LockingSchraube über das 2. Gewinde am Schraubenkopf winkelstabil in der Platte verriegelt wird [50].

\section{Kompressionsplatten}

Die erste Osteosynthese mit Kompressionsplatten im maxillofazialen Bereich wurde bei einem Patienten mit bilateralen Frakturen im hochatrophen Unterkieferkorpus (Klasse III) durchgeführt (OP: Hans-Georg Luhr Oktober 1967, Hamburg-Eppendorf - siehe [51).

Unkontrollierbare sekundäre Dislokationseffekte, unzureichende interfragmentäre Abstützungsmöglichkeiten im atrophen Knochen und die nur zu einem Load Sharing geeigneten Plattenquerschnitte und -längen gehören $\mathrm{zu}$ den Gründen, weshalb Kompressionsplatten - trotz anfänglicher Erfolgsmitteilungen $[11,12,52,53]$ - schnell aus dem allgemeinen Behandlungsrepertoire bei Frakturen im zahnlosen Unterkiefer verschwunden sind und heute als „nicht mehr empfehlenswert" apostrophiert werden [41].

\section{Pencilbone Plate}

Die sog. „Pencilbone Plate“ ist eine speziell für die Frakturversorgung im atrophen Unterkiefer entwickelte Osteosyntheseplatte (Namensgebung bezieht sich auf den oftmals nur noch bleistiftdünnen atrophen Knochen, vgl. [54] - Abb.5a und b) und soll den besonderen biomechanischen und biologischen Anfor- derungen besser gerecht werden [55, 56].

Bei diesem Plattentyp sollten anfangs die Eigenschaften von Miniplattensystemen, also insbesondere die einfache technische Handhabung, die eine transorale Applikation erlaubt, mit den möglichen Vorteilen von Kompressionseffekten vereint werden (Abb. 5c).

Die erste Version dieser Platten besaß einen oval geformten und verstärkten Mittelsteg zur Stabilisierung des frakturnahen Knochenbereichs und hatte 2 sphärische Gleitlöcher jenseits der Fraktur. An den verstärkten Teil der Osteosyntheseplatte schlossen sich jeweils 2 bzw. 3 normale unverstärkte Löcher an, die sich sehr leicht an den frakturfernen Knochenbereich adaptieren ließen [55]. Das Design und die Dimensionen der Pencilbone-Platte sind auch in einer neuen abgeänderten Designvariante (Abb.5d) grazil geblieben und sollen eine genaue Adaptation an die Knochenoberfläche über längere Strecken ermöglichen.

Die für Pencilbone-Platten experimentell ermittelten Belastungswerte im Frakturspalt sollen eine primäre Knochenheilung günstig beeinflussen (Blume et al. 2003), sofern die interfragmentäre Abstützung noch ausreichend ist. Ein Load Bearing ist a priori nur mit Rekonstruktionssystemen möglich [57].

\section{Synthes UniLOCK 2.0 ${ }^{\mathrm{TM}}$}

Vor über 10 Jahren kam ein unidirektionales, winkelstabiles Mini-Locking-Plattensystem (Synthes UniLOCK 2.0 ${ }^{\mathrm{TM}}$ ) auf den Markt, das in 3 Profilstärken (Large Profile, Medium Profile, Small Profile) erhältlich ist. Diese 3 Plattenvarianten ergänzen die 2.4-UniLOCK-Rekonstruktionsplatte zu einer Locking-Platten-Familie im Sinne eines „Downsizing“ nach unten.

Die Dimensionierung der Small Profile UniLOCK-Platte entspricht einer konventionellen Miniplatte, während die Medium und Large Profile-Platten Materialquerschnitte (Dicke und Breite) aufweisen, die ihre Biegestabilität stufenweise in die Nähe der 2.4-Universal-FrakturenPlatte bzw. der 2.4-Locking-Rekonstruktionsplatte rücken (Abb.6). Demnach werden Platten mit diesen Profilen als Intermediärplatten bezeichnet.

In Verbindung mit revaskularisierten Knochentransfers zum Unterkieferersatz 

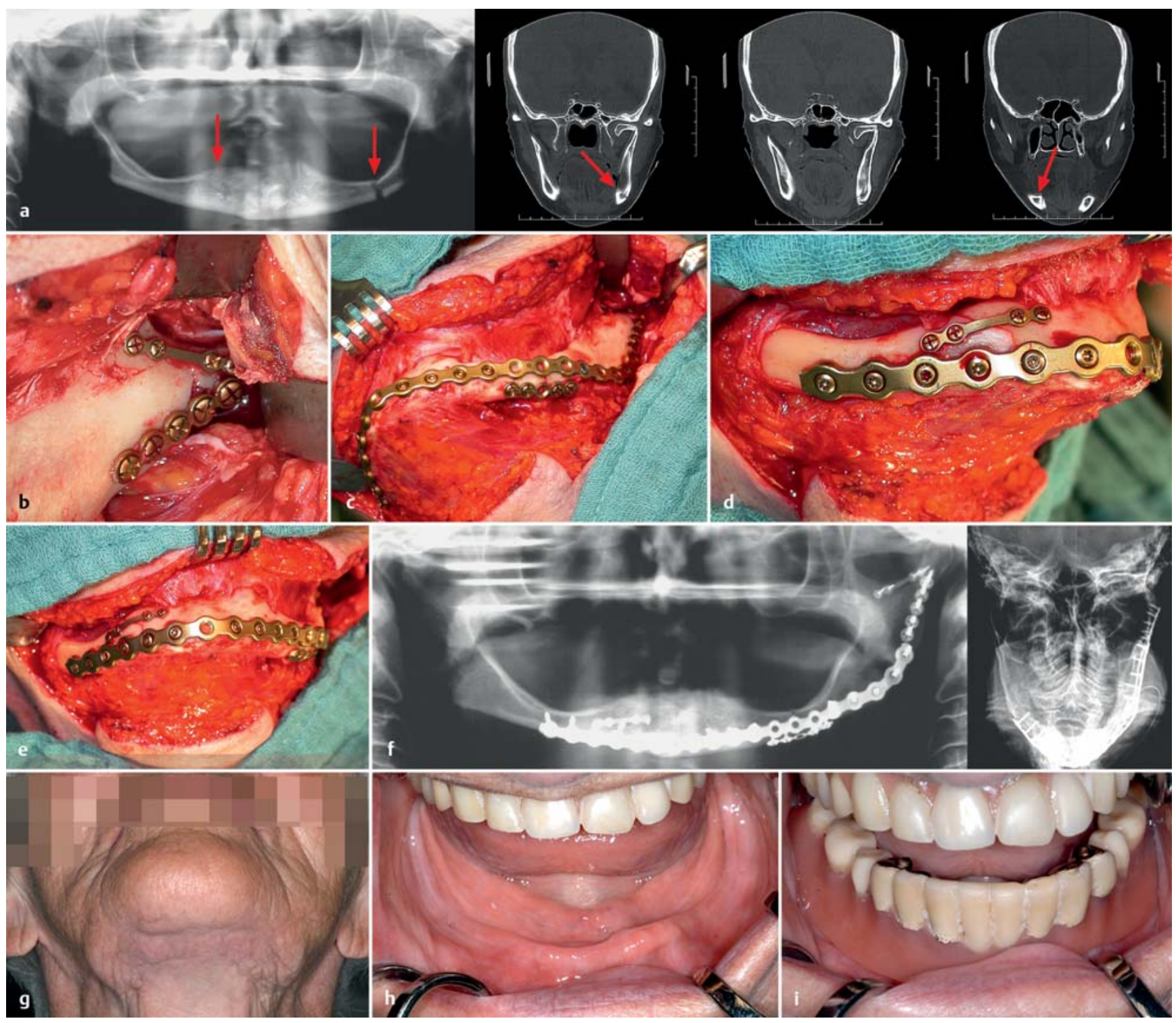

Abb. $\mathbf{6}$ a bis i Versorgung einer atrophen Unterkiefer-3-fach-Fraktur, Luhr-Klasse II im Sinne eines „Downsizing“ (80 Jahre alte Patientin nach Treppensturz). a Präoperative Panoramaschichtaufnahme (PSA) und koronare CT-Schichten - Pfeile zeigen auf die Frakturstellen in der Korpusmitte links, der Prämolarenregion rechts und an der Kiefergelenkfortsatzbasis links - Luxation des Kieferköpfchens; b Beginn mit Reposition und Fixation des Kiefergelenkfortsatzes links; c-e intermediäre Large-Profile-UniLOCK-2.0-Platte zur Überbrückung vom Kieferwinkelbereich links bis zum Unterkieferkorpus rechts, nach Vorfixierung mit Adaptationsplatten basal im Korpusbereich links und lateral oben paramedian rechts; $\mathbf{f}$ Postoperative PSA und postoperatives okzipitomentales Schädel-Röntgenbild; $\mathbf{g}$ und $\mathbf{h}$ Narben submandibuär und submental nach Zugang im Zickzackmuster; i wiederhergestelltes Prothesenlager im Unterkiefer und eingesetzte frühere Prothese.

ist bereits verschiedentlich über den erfolgreichen Einsatz dieser „kleinen“ Rekonstruktionsplatten berichtet worden $[58,59]$. Obwohl im Werbematerial die Eignung der „Medium“ und v.a. die „Large Profile“-Platten zur Osteosynthese von Frakturen im zahnlosen atrophen Unterkiefer besonders hervorgehoben wurde, ist in bisherigen Veröffentlichungen nur anhand von Einzelbeispielen $[42,47,49,60]$ und einer ersten kleinen Vergleichsstudie [61] auf die möglichen Vorteile dieser Plattenformate hingewiesen worden. Gegenüber den 2.4Locking-Rekonstruktionsplatten verein- fachen sich die Biegbarkeit und die Ankonturierung. Die reduzierten Profile verringern die Palpierbarkeit und die Perforationsgefahr durch die Weichgewebe. Interferenzen zwischen Platte und Prothesensattel werden vermieden, sofern eine kaufunktionelle Rehabilitation beabsichtigt ist.

\section{Knochenaugmentation}

Zur Sicherstellung einer primären Knochenheilung wurden bei Frakturen im hochatrophen Unterkiefer seit Langem Knochenersatzplastiken entweder allein oder simultan mit Plattenosteosynthesen verwendet (z.B. [62]) (Abb. 7). In Verbindung mit modernen Locking-Plattensystemen haben ossäre Soforttransplantate nicht nur eine Förderung der Frakturkonsolidierung durch Einbringen osteokompetenter Zellen [43,49,63], sondern auch die Augmentation der Alveolarfortsätze für eine spätere implantologisch-prothetische Versorgung zum Zweck [42]. Die Bedarfslage nach einer kaufunktionellen Rehabilitation variiert bei Patienten mit Frakturen im hochatrophen Unterkiefer nach Alter, psychosozialen Gegebenheiten, finanziellen 


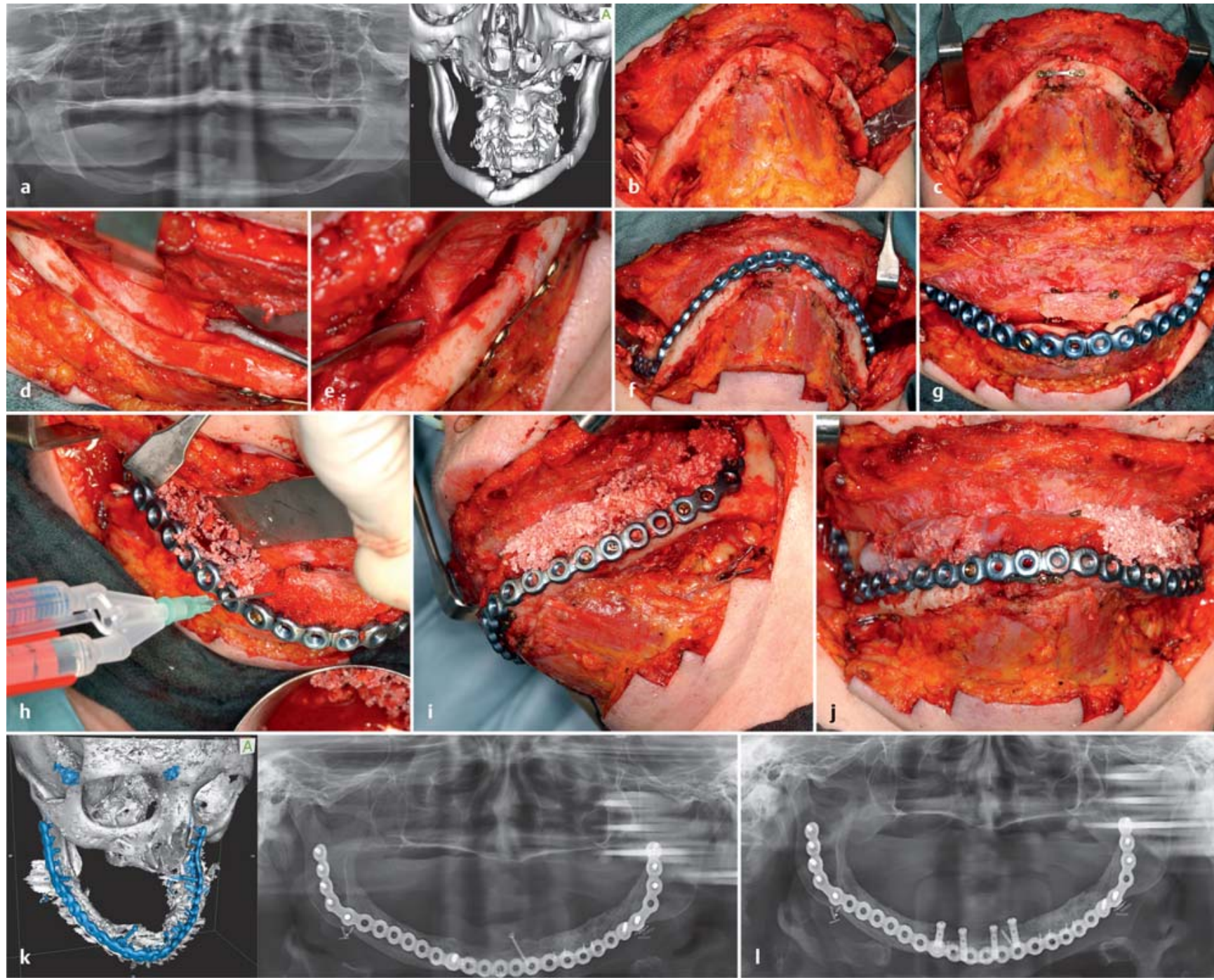

Abb. 7 a bis I Knochenaugmentation im Rahmen der primären Versorgung einer Zweifachfraktur im atrophen zahnlosen Unterkiefer (Luhr-Klasse III - 67-jährige Patientin). a1 und a2 Präoperative PSA und präoperatives 3-D-CT; b Freilegung der gesamten basalen Unterkieferspange; c Vorfixierung der mehrfragmentären Fraktur paramedian rechts sowie im Unterkieferkorpus links mit Mini-Adaptationsplatten; d und e Abheben des krestalen Mukoperiosts über dem Korpusbereich bds. - wannenförmige Atrophie und R. incisivus, in den anterior erhaltenen Knochenkanal einstrahlend; $\mathbf{f}$ rigide Fixation mittels Rekonstruktionsplatte. $\mathbf{g}$ Mit Schrauben schwebend fixiertes kortikospongiöses Beckenkammtransplantat anterior; $\mathbf{h}$-j Augmentation im Korpusbereich bds. und vorne mit Beckenkammspongiosa pur bzw. einem Gemisch mit Bio Oss; k1 und k2 Postoperatives 3-D-CT mit kolorierter Rekonstruktionsplatte und PSA 4 Monate postop. - Schraube vorne rechts wurde entfernt; I PSA nach Insertion von 4 Implantaten.

Möglichkeiten, usw. und wird relativ selten nachgefragt, wenn vor dem Trauma keine Vollprothesen zum Zahnersatz getragen wurden.

Für den Fall einer Frakturüberbrückung in Kombination mit einem Knochenaufbau als präprothetisch-/präimplantologische Maßnahme werden Adaptionsplatten in Intermediärformaten favorisiert, da sich bei diesen Metallentfernungen wegen der geringeren Profilhöhe („low profile“) im Gegensatz zu Makro-/ Rekonstruktionsplatten erübrigen sollen $[42,64]$.

\section{Operativer Zugang}

Je nach Typ und Größe der Hardware kommen für die offene Reposition und interne Fixation (ORIF) transorale oder externe operative Zugangswege infrage. Die Freilegung des zahnlosen atrophen Unterkiefers über externe submandibuläre Schnittführungen geht mit dem Risiko von Läsionen des R. marginalis mandibulae (VII) einher, erlaubt andererseits aber eine übersichtliche Darstellung sämtlicher Unterkieferabschnitte, falls nötig bis in den Bereich der Gelenkfortsätze.
Vorteilhaft sind Zickzackinzisionen der Haut in direkter Nähe bzw. unterhalb der Mandibula unter Ausnutzung vorhandener Hautfalten (Abb.6, 7, 9). Nach Ablösen eines Hautlappens in kaudaler Richtung wird dann auf gewohnter Höhe ein Wechselschnitt durch das Platysma vorgenommen.

Die Zickzackschnittführung ist topografisch weniger ausgedehnt als eine kontinuierliche Inzision und lässt sich aufgrund ihrer Geometrie aufdehnen wie eine Ziehharmonika. Die Elastizität der Cutis, welche die Aufdehnung von Wundkavitäten limitiert, wird somit trickreich außer Kraft gesetzt. 
Transorale Zugänge in der Schleimhaut des anterolateralen Vestibulums eignen sich gut zur Exposition der Kinn- und seitlichen Unterkieferkorpus-Region.

Der Hauptvorteil transoraler Zugänge besteht darin, dass im Gesicht keine äußerlich sichtbaren Narben entstehen. Das Risiko einer Verletzung des N. mentalis oder buccalis sowie ein „Drooping chin“ (hängendes Kinn) als Folge einer unzureichenden Readaptation der Mentalismuskulatur sind hingegen nicht gänzlich ausgeschlossen. Falls die Knochenatrophie weit fortgeschritten ist, können sowohl der N. alveolaris als auch die zugehörige Arterie [1] frei unter dem Mukoperiost liegen und bei Inzision und Präparation direkt verletzt werden [42].

\section{Therapieverfahren und ihre Komplikationen}

$\mathrm{Zu}$ den typischen Lokalkomplikationen in der Behandlung von Frakturen des zahnlosen atrophen Unterkiefers gehören Infektion, Sequestrierung kleinerer Fragmente, Osteomyelitis, freiliegende Platten, Fehlstellung der Fragmente, Pseudarthrose bzw. Non-Union [65], Schraubenlockerungen, Schraubenausrisse und Ermüdungsbrüche der Osteosyntheseplatten. Es wird über hohe Komplikationsraten in Größenordnungen von 4 bis zu 20\% (bezogen auf Zahl der Patienten oder Anzahl der Frakturen) berichtet (z.B. $[2,3,40,42,53,66])$, wobei regelmäßig Korrelationen mit den folgenden (z.T. schon vorangehend angesprochenen) Aspekten und Problemkreisen hergestellt werden [42]:

- Atrophiegrad/-klasse

- geschlossene Behandlung oder offene Reposition und Fixation

- operative Zugangswege: intraoral vs. extraoral vs. Kombination aus beiden

- Deperiostierung: subperiostale vs. supraperiostale Präparation und Plattenapplikation

- Hardware-Dimension und HardwareTyp bei interner Fixierung: Miniplatten vs. Intermediär- vs. Makrobzw. Rekonstruktionsplatten, Kompressionsplatten, Spezialplatten, Locking- vs. Non-Locking-Platten

Nicht zu unterschätzen sind die auf die atrophe Unterkieferspange einwirkenden Zugkräfte durch die antagonistischen Muskelgruppen - Kaumuskulatur einerseits (Masseter-PterygoideusSchlinge und $\mathrm{Mm}$. temporales) und die suprahyoidale Muskulatur andererseits (Abb. 8).

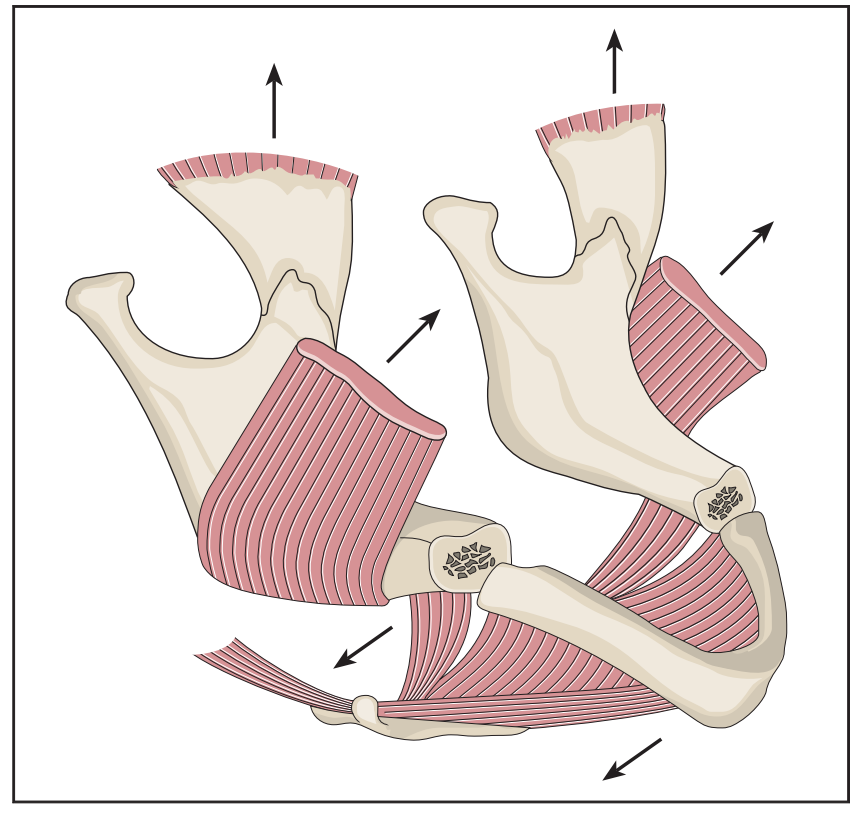

Abb. 8 Dislozierende Zugwirkung auf den atrophen, bilateral im Korpus frakturierten Unterkiefer durch die Kaumuskulatur und suprahyoidale Muskulatur.
Bei bilateralen Frakturen im Korpusbereich sind sie als Risikofaktor für Dislokationen zu werten und müssen bei der Auswahl des Osteosynthesematerials berücksichtigt werden. Dislokationen des vorderen Unterkieferbogens sind kein seltener klinischer Initialbefund und immer von einer Atemwegsbehinderung begleitet, weil die Aufhängung der Zunge am Hyoid kompromittiert ist.

\section{Hardware-„Downsizing“ möglich?}

Die Indikation zur Verwendung eines Plattensystems ist letztendlich an der Restknochenhöhe und der Knochenqualität zu orientieren. Welche Plattengrößen, Plattenprofile, Adaptation, Kompression, interner Fixateur zur Versorgung von Frakturen im zahnlosen atrophen Unterkiefer geeignet sind, steht allerdings nach wie vor zur Debatte.

Mit Blick auf „minimalinvasive Vorgehensweisen“ geht der Trend bei der Behandlung von Unterkieferfrakturen ganz allgemein zu einem „Downsizing“ der Osteosynthese-Hardware (Abb.6). Welche Plattenformate in Anzahl und Konfiguration, welches Applikationsprinzip (Kompression, Adaptation, Fixateur interne) oder welche Schraubentypen (Non-Locking, Locking) und Schraubendurchmesser sich zur Dimensionsreduzierung des Osteosynthesematerials bei der Frakturversorgung im zahnlosen atrophen Unterkiefer eignen, ist bisher weder im direkten klinischen Vergleich noch experimentell hinreichend geklärt [67].
In einer eigenen, bisher unveröffentlichten In-vitro-Studie wurden die Stabilität und die Handling-Eigenschaften moderner Hardware-Systeme in Mini- und Intermediärformaten im Vergleich zu Rekonstruktionsplatten in konventioneller und winkelstabiler Montage als Goldstandard bzw. Kontrolle überprüft.

Bei geringgradig atrophierten Unterkiefern (Luhr-Klasse I) erscheint ein „Downsizing“ mit einer Auswahl von Plattensystemen (z. B. Intermediärplatten, Pencilbone-Platten oder multiplen Platten in 3-D-Anordnung) grundsätzlich möglich.

Ob dies auf höhergradige Atrophiegrade übertragbar ist, bedarf weiterer Untersuchungen, erscheint jedoch zumindest für Miniplatten und die Medium-Profile-Plattenvarianten fragwürdig $[63,68$, 69].

\section{Ausblick - Alternative Plattenposi- tionierung: Basal and Lingual Plating}

Die Perforationsgefahr durch das Weichgewebe ist ein wesentlicher Nachteil großer Plattenprofile. Interferenzen zwischen prominenten Plattenanteilen im anterolatealen Unterkiefervestibulum und Prothesensattel erschweren oder verhindern sogar eine kaufunktionelle Rehabilitation.

Diese Schwierigkeiten könnten mit der Applikation von 2.4 (oder größeren) Locking-Rekonstruktionsplatten am Basalrand des Unterkiefers („Basal Plating“) 

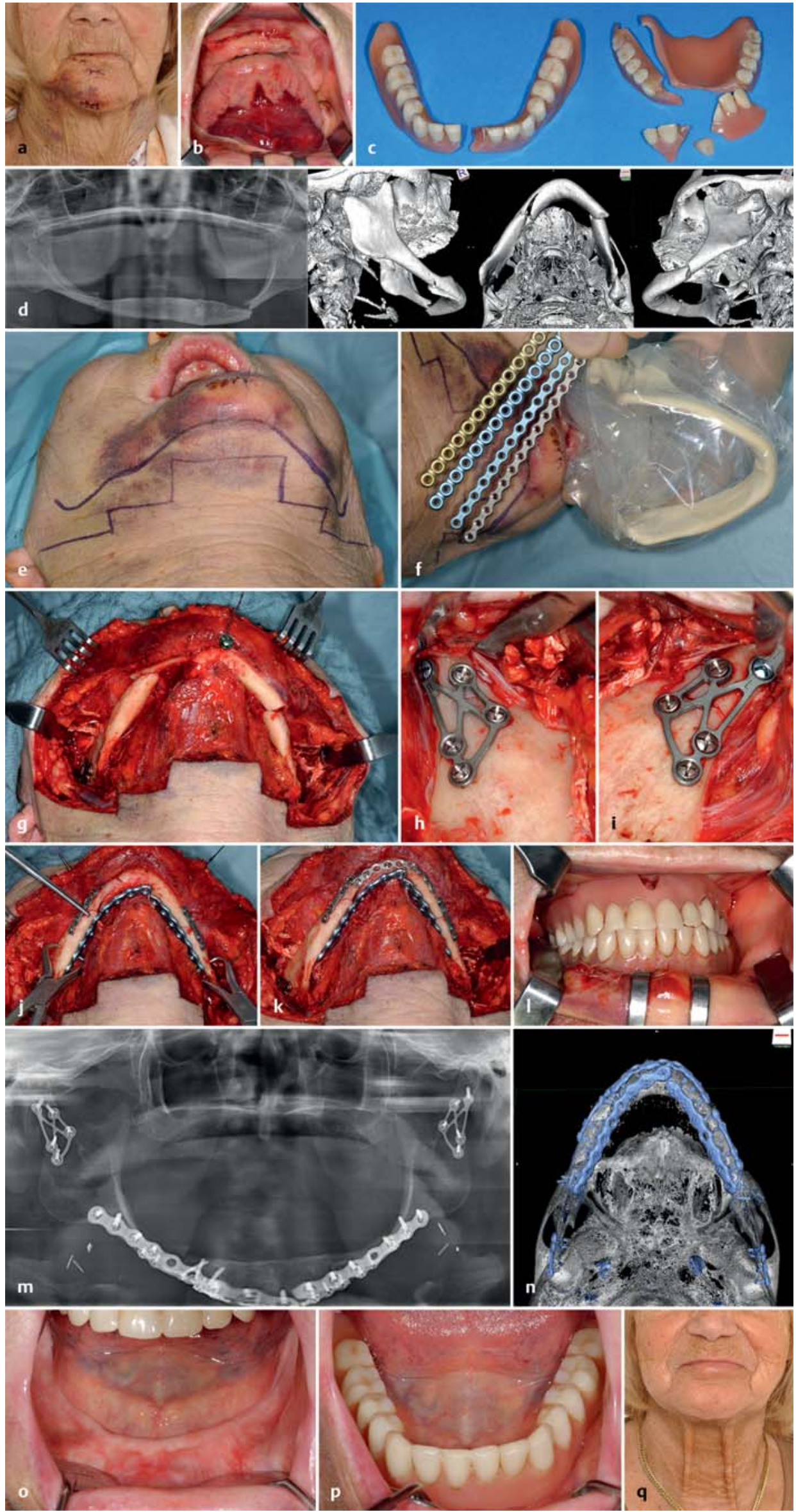

Abb. 9a bis q Mehrfachfraktur bei zahnlosem, hoch atrophem Unterkiefer (Luhr-Klasse III, Patientin 77 Jahre). a Hämatome und Platzwunde unter dem Kinn; b Massive Unterblutung von Mundboden- und SublingualSchleimhaut; $\mathbf{c} \mathbf{1}$ und $\mathbf{c} \mathbf{2}$ beim Sturz zerbrochene Ober- und UnterkieferVollprothesen; d1-d4 Präoperatives PSA und 3-D-CTs - Frakturmuster: Kiefergelenkortsatzbasisfraktur bds., links mit Lateral Override, rechts mit medialer Achsknickung, Unterkieferkorpusfrakturen bds., mehrfragmentär rechts; e submandibulärer OP-Zugang Zickzack- bzw. Stufenpyramiden Schnittführung; f Auswahl an Rekonstruktionsplatten (Matrix Mandible Recon) und zahnloses Unterkiefermodell in Sterilfolie zur Plattenkonturierung; $g$ weite Exposition des Unterkiefers bis in die Gelenkfortsatzregion bds.; $\mathbf{h}$ und $\mathbf{i}$ Reposition und Fixation der Kiefergelenkfortsätze bds.; j Fragmentreposition und Vorfixierung der Unterkieferspange über Miniadaptationsplatten bds. lateral am Korpus sowie Anpassen des „Lingual Plating“; k linguale Platten (Plattendicke $2 \mathrm{~mm}$ Large Profile Unilock 2.0) fixiert, komplettierte Osteosynthesen ventral über Symphysen-/Kinnregion; I Reparierte und am OP-Ende eingesetzte Vollprothesen mit ungestörtem Sitz und guter Okklusion; $\mathbf{m}$ und $\mathbf{n}$ postoperative PSA und 3-D-CT von basal mit koloriertem Osteosynthesematerial; $\mathbf{o}$ und $\mathbf{p}$ intraorale Situation und eingesetzte Prothesen 6 Monate postoperativ; q Narbenverhältnisse submental/submandibulär. 


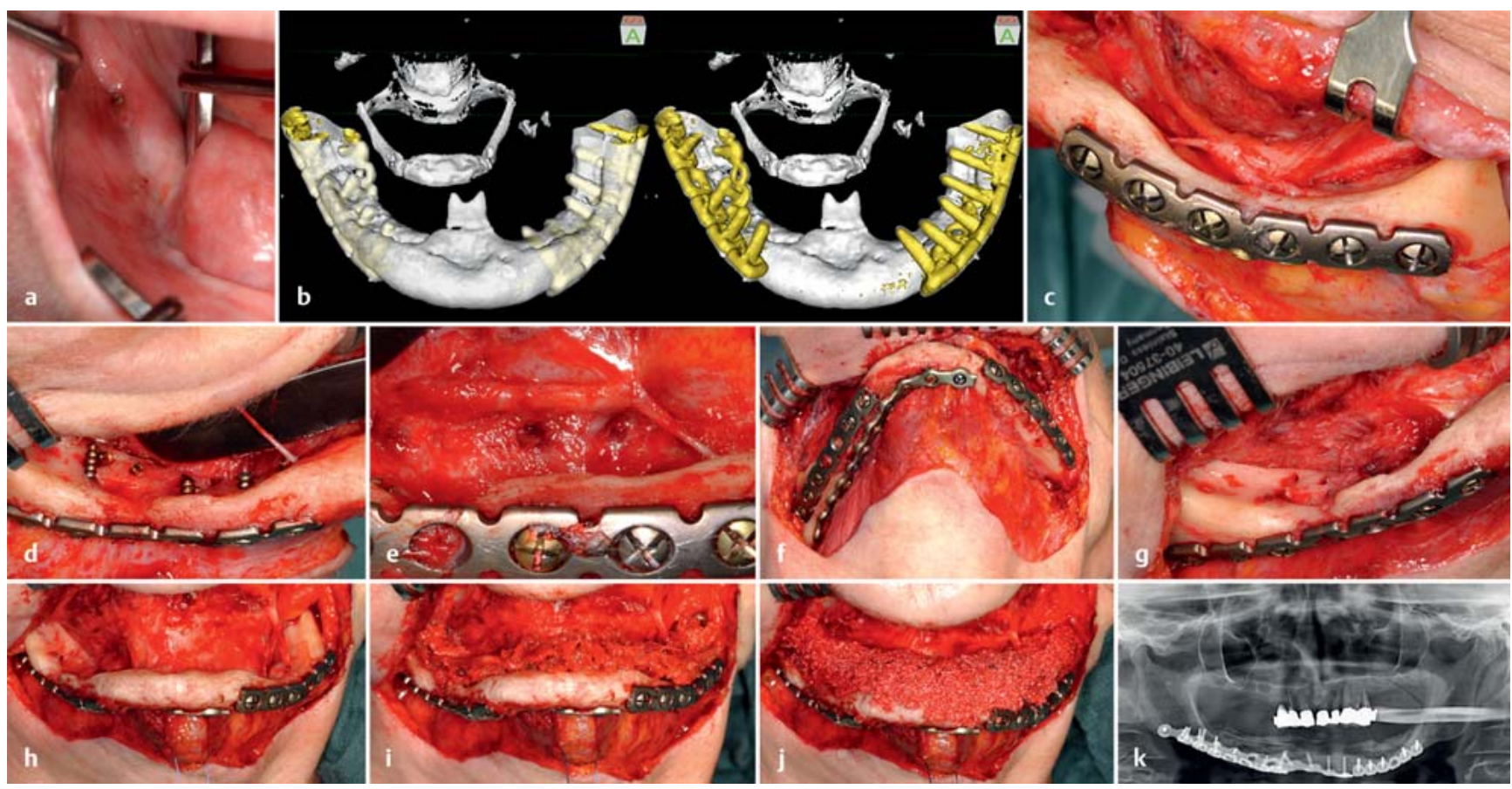

Abb. 10 a bis k „Salvage Procedure“ nach rigider Fixierung einer bilateralen Unterkieferkorpusfraktur mit „Lingual Plating“ (Luhr-Klasse III, Patientin 77 Jahre). Infolge progredienter Atrophie des Alveolarfortsatzes liegen die von der Lateralfläche eingebrachten Schrauben in der krestalen Knochenwanne frei, fragliche Knochenkontinuität rechts. a Intraoral sichtbare vestibuläre Schleimhautperforation dorsal rechts; b1 und b2 Osteosynthesematerial im 3-D-CT in 2 Transparenzstufen - rechts Perforation mehrerer Schrauben, deren Spitzen bereits früher abgeschliffen worden sind; c krestale Knochenwanne links - der N. alveolaris inf. und seine Verzweigung in den N. mentalis und den R. incisivus (nach intraossär) sind sichtbar; d krestale Wanne rechts mit freiliegenden Schrauben von lateral und kaudal - vorne der R. incisivus; e Verzweigungen des N. alveolaris inf. rechts mit Bestätigung, dass es sich um den R. incisivus handelt; $\mathbf{f}$ Applikation einer Large Profile UniLock 2.0-Platte von lingual nach Platten- bzw. Schraubenentfernung bzw. Austausch; g krestale Wanne rechts ohne perforierende Schrauben; h-j Aufbau des Unterkieferalveolarfortsatzes mit Spongiosa vom posterioren Beckenkamm und einer Bio Oss Layer on Top; $\mathbf{k}$ postoperative PSA.

über einen extraoralen Zugang gelöst werden [70,71]. Die Lateralflächen des Unterkiefers werden zum Anbringen der Platte nur wenig freigelegt und die Positionierung der Platte tangiert die dünne oralwärts lokalisierte Weichgewebebedeckung (Mukoperiost und adhärente Gingiva) von vorneherein nicht mehr. Infolge dieser vorteilhaften Integration in den Weichgewebsmantel sinkt die Wahrscheinlichkeit für Wunddehiszenzen und eine Plattenexposition. Da die krestal und beidseits davon gelegenen Knochenoberflächen des Unterkiefers ausgespart bleiben, steht die Platte den Kunststoffsätteln einer schleimhautgetragenen Prothese nicht im Wege. Nachteilig bei dieser Vorgehensweise sind die senkrecht von unten eingebrachten Schrauben, die den Knochen nur monokortikal fassen dürfen, damit ihre Spitzen nicht unter der Schleimhaut an der Prothesenbasis zu liegen kommen.

In einer In-vitro-Testreihe an atrophen Kunststoffunterkiefern ergaben sich keine Unterschiede im mechanischen Verhalten zwischen Rekonstruktionsplatten, die am Basalrand des Unterkiefers
(„Inferior border fixation“) oder auf übliche Weise entlang der Lateralfläche („Lateral border fixation“) angebracht werden [71].

Technisch nicht ganz einfach gestalten dürfte sich indessen das Anbiegen der kräftigen Rekonstruktionsplatten über die Kante, insbesondere, wenn die Platte bei einer schmalen Kinn-/Unterkiefersymphysenregion in die Form einer Parabel gebracht werden muss.

Eine weitere Option zur Plattenapplikation besteht in der Positionierung auf der Lingualseite des Unterkieferrands („Lingual Plating“) (Abb. 9) Dabei bleibt das Unterkiefervestibulum ebenfalls für einen Prothesensattel frei, sodass eine sofortige Prothesenfähigkeit garantiert ist.

Beim Lingual Plating wird das Knochenangebot im atrophen Unterkiefer vorteilhaft ausgenutzt: auf der Lingualseite der Unterkieferbasis, unterhalb der Linea mylohyoidea, bleiben die Knochenverhältnisse konstant und sind kaum von Resorptionsvorgängen betroffen (Metzger unveröffentlicht). Zugleich schreitet die Atrophie des Alveolarfortsatzes in der Etage darüber von lingual nach vestibuär fort und hinterlässt eine hohe $\mathrm{Au}$ ßenkante vestibulär. Häufig wird dabei der ehemalige Krestalbereich wannenoder trogförmig ausgekehlt (vgl. Abb. 7d und e). Insgesamt nimmt der Unterkieferkorpus einen triangulären Querschnitt an, in dem - in Schrägrichtung von unten innen nach oben außen eingebracht - Schrauben größerer Länge verwendet werden können als bei Insertion von der Lateralfäche aus. Die Schleimhaut- bzw. Mukoperiostbedeckung im Krestalbereich ist zudem einem geringeren Perforationsrisiko ausgesetzt.

Biomechanische Aspekte bei der lingualen Plattenapplikation sind derzeit noch nicht näher untersucht, dürften aber näherungsweise ähnlich einzuschätzen sein wie beim „Basal Plating“.

Ein Lingual Plating kommt auch als „Salvage“ infrage, wenn es nach der Applikation rigider Osteosyntheseplatten von der Lateralseite zur weiteren krestalen Atrophie gekommen ist und Schraubenschäfte und -spitzen nach medial unter oder durch die Schleimhaut ragen. 
Da die Platten belassen werden müssen, um die Stabilität beim hochatrophen Unterkiefer nicht zu gefährden, wird empfohlen, die freiliegenden Schraubenpartien über einen intraoralen Zugang abzufräsen [72]. Falls die Situation wiederholt auftritt oder mehrere Schrauben nach lingual vorstehen und entfernt werden müssen, bietet sich eine linguale Platteninsertion an, um das Konstrukt zu retten (Abb. 10). Das Lingual Plating übernimmt die Stabilisierung und dient als Protektion, bis ein Knochentransplantat (bspw. autologe Spongiosa in Kombination mit xenogenem Bio Oss) eingeheilt ist und zur Verfestigung des Unterkiefers geführt hat.

\section{Fazit}

Die Indikation zur Verwendung eines Plattensystems bei Frakturen im zahnlosen atrophen Unterkiefer ist an der Restknochenhöhe zu orientieren. Experimentell kommen kleindimensionierte Plattensysteme schon bei der Luhr-Klasse I nur bedingt infrage.

Mit Abnahme der Vertikaldimension auf die Luhr-Klasse III wird sich trotz neuer Plattensysteme wahrscheinlich die vertraute Formel bewahrheiten: je niedriger die Restknochenhöhe, desto stärker und länger die zu verwendende Osteosyntheseplatte.

\section{Literatur}

${ }^{1}$ Bruce RA, Strachan DS. Fractures of the edentulous mandible: the Chalmer J. Lyons Academy study. J Oral Surg 1976; 34: 973-979

2 Thaller SR. Fractures of the edentulous mandible: a retrospective review. J Craniofac Surg 1993; 4: 91-94

${ }^{3}$ Amaratunga NA. A comparative study of the clinical aspects of edentulous and dentulous mandibular fractures. J Oral Maxillofac Surg 1988; 46: 3-5

${ }^{4}$ Kunz C, Hammer B, Prein J. [Fractures of the edentulous atrophic mandible. Fracture management and complications]. Mund Kiefer Gesichtschir 2001; 5: 227-232

5 Bruce RA, Ellis E 3rd. The second Chalmers J. Lyons Academy study of fracture of the edentulous mandible. J Oral Maxillofac 1993; 51: 904-911

${ }^{6}$ Xie Q Ainamo A. Association of edentulousness with systemic factors in elderly people living at home. Community Dent Oral Epidemiol 1999; 27: 202-229

${ }^{7}$ McGregor AD, MacDonald DG. Age changes in the human inferior alveolar artery-a histological study. Br J Oral Maxillofac Surg 1989; 27: 371-374

${ }^{8}$ Bradley JC. Age changes in the vascular supply of the mandible. Br Dent J 1972; 132: 142144

${ }^{9}$ Bradley JC. Age changes of the inferior alveolar artery. Br J Oral Maxillofac Surg 1988; 26 : 517-519
${ }^{10}$ Härle F. Atlas der präprothetischen Operationen. München: Hanser Verlag; 1989

${ }^{11}$ Luhr HG, Reidick T, Merten HA. Fractures of the atrophic mandible-a challenge for therapy. Fortschr Kiefer Gesichtschir 1996a; 41: 151-154

12 Luhr HG, Reidick T, Merten HA. Results of treatment of fractures of the atrophic edentulous mandible by compression plating: a retrospective evaluation of 84 consecutive cases. J Oral Maxillofac Surg 1996b; 54: 250-254

13 Carlsson GE, Ragnarson N, Astrand P. Changes in height of the alveolar process in edentulous segments. A longitudinal clinical and radiographic study of full upper denture cases with residual lower anteriors. Odontol Tidskr 1967; 75: 193-208

14 Wical KE, Swoope CC. Studies of residual ridge resorption. I. Use of panoramic radiographs for evaluation and classification of mandibular resorption. J Prosthet Dent 1974; 32: 7-12

15 Atwood DA. Bone loss of edentulous alveolar ridges. J Periodontol 1979; 50: 11-21

${ }^{16}$ Cawood JI, Howell RA. A classification of the edentulous jaws. Int J Oral Maxillofac Surg 1988; 17: 232-236

17 Nasser M, Fedorowicz Z, Ebadifir A. Management of the fractured edentulous atrophic mandible. Cochrane Database of Systematic Review 2007; 1: CD006087

18 Cope MR. Spontaneous fracture of an atrophic endentulous mandible treated without fixation. Br J Oral Surg 1982; 20: 22-30

19 Wolfe SA, Lovaas M, McCafferty LR. Use of a miniplate to provide intermaxillary fixation in the edentulous patient. J Craniomaxillofac Surg 1989; 17: 31-33

${ }^{20}$ Hori M, Nakada Y, Matsunaga S et al. Use of two miniplates for intermaxillary skeletal fixation in the treatment of jaw deformity and fracture. J Nihon Univ Sch Dent 1992; 34: 224-229

21 Pohl L. Extraoral splinting of the edentulous mandible. Lancet $1941 ; 2: 389-391$

22 Ginestet G. Le "fixateur externe“ dans le traitement des fractures du maxillaire inférieur. Rev Odontol Stomatol Maxillo-Fac 1946; 10: 455-460

${ }^{23}$ Kaufman Y, Cole P, Hollier L. Contemporary issues in facial gunshot wound management. J Craniofac Surg 2008; 19: 421-427

24 Alpert B, Tiwana PS, Kushner GM. Management of comminuted fractures in the mandible. Oral Maxillofac Surg Clin North Am 2009; 21: 185-192

${ }^{25}$ Madsen MJ, Haug RH, Christensen BS et al. Management of atrophic mandible fractures. Oral Maxillofac Surg Clin North Am 2009; 21: 175-183

${ }^{26}$ Ellis E 3rd, Miles BA. Fractures of the mandible: a technical perspective. Plast Reconstr Surg 2007; 120: 76S-89S

27 Michelet FX, Dessus B, Benoit JP et al. [Mandibular osteosynthesis without blocking by screwed miniature stellite plates]. Rev Stomatol Chir Maxillofac 1973; 74: 239-245

${ }^{28}$ Champy M, Wilk A, Schneebelen JM. Die Behandlung der Mandibularfrakturen mittels Osteosynthese ohne intermaxilläre Ruhigstellung nach der Technik von F.X. Michelet. Zahn Mund Kieferheilk 1975; 63: 339-341

29 Champy M, Lodde JP, Jaeger JH et al. [Biomechanical basis of mandibular osteosynthesis according to the F.X. Michelet method]. Rev Stomatol Chir Maxillofac 1976a; 77: 248251

30 Champy M, Lodde JP, Jaeger JH et al. [Mandibular osteosynthesis according to the Michelet technic. I. Biomechanical bases]. Rev Stomatol Chir Maxillofac 1976b; 77: 569-576
31 Champy M, Lodde JP, Jaeger JH et al. [Mandibular osteosynthesis according to the Michelet technic. II. Presentation of new material. Results]. Rev Stomatol Chir Maxillofac 1976c; 77: $577-582$

32 Champy M, Lodde JP, Jaeger JH et al. [Mandibular osteosynthesis according to the Michelet technic. Justification of new material. Results]. Rev Stomatol Chir Maxillofac 1976d; 77: 252-255

${ }^{33}$ Iatrou I, Samaras C. Theologie-Lygidakis N, Miniplate osteosynthesis for fractures of the edentulous mandible: a clinical study 198996. J Craniomaxillofac Surg 1998; 26: 400 404

${ }^{34}$ Choi BH, Huh JY, Suh CH et al. An in vitro evaluation of miniplate fixation techniques for fractures of the atrophic edentulous mandible. Int J Oral Maxillofac Surg 2005; 34: 174 177

35 Mugino H, Takagi S, Oya R et al. Miniplate osteosynthesis of fractures of the edentulous mandible. Clin Oral Investig 2005; 9: 266270

${ }^{36}$ Pape HD, Gerlach KL, Champy M. Mandibular Fractures including atrophied Mandible. In: Härle F, Champy M, Terry BC, eds. Atlas of craniomaxillofacial Osteosynthesis. Miniplates, Microplates and Screws. Stuttgart, New York: Thieme; 1999: 31-39

37 Spiessl B, Schargus G, Schroll K. Die stabile Osteosynthese bei Frakturen des unbezahnten Unterkiefers. Schweiz Monatschr Zahnheilk 1971: 81: 39-45

38 Spiessl B. Osteosynthese des Unterkiefers, Manual der AO-Prinzipien. Berlin, Heidelberg, New York: Springer Verlag; 1988

39 Prein J. Invited Comment to Newman L: The role of autogenous rib grafts in treating fractures of the atrophic edentulous mandible. $\mathrm{Br}$ J Oral Maxillofac Surg 1990; 33: 386

40 Eyrich GK, Grätz KW, Sailer HF. Surgical treatment of fractures of the edentulous mandible. J Oral Maxillofac Surg 1997; 55: 10811087; discussion 1087-1088

${ }^{41}$ Schilli $W$, Stoll P, Bähr $W$ et al. Mandibular Fractures. In: Prein J, ed. Manual of internal Fixation in the cranio-facial Skeleton. Berlin Heidelberg, New York: Springer; 1998: 86-87

42 Ellis $E$ 3rd, Price C. Treatment protocol for fractures of the atrophic mandible. J Oral Maxillofac Surg 2008; 66: 421-435

43 Tiwana PS, Abraham MS, Kushner GM et al. Management of atrophic edentulous mandibular fractures: the case for primary reconstruction with immediate bone grafting. J Oral Maxillofac Surg 2009; 67: 882-887

44 Haug RH, Street CC, Goltz M. Does plate adaptation affect stability? A biomechanical comparison of locking and nonlocking plates. J Oral Maxillofac Surg 2002; 60: 1319-1326

45 Prein J, Rahn B. Scientific and technical Background. In: Prein J, ed. Manual of internal Fixation in the cranio-facial Skeleton. Berlin, Heidelberg New York: Springer; 1998: 9

${ }^{46}$ Gutwald R, Büscher P, Schramm A. Biomechanical stability of an internal mini-fixation-system in maxillofacial osteosynthesis Med Biol Eng Comp 1999; 37 (Suppl. 2): 280

47 Gutwald R, Alpert B, Schmelzeisen R. Principle and stability of locking plates. Keio J Med 2003; 52: 21-24

48 Sikes jr. JW, Smith BR, Mukherjee DP. An in vitro study of the effect of bony buttressing on fixation strength of a fractured atrophic edentulous mandible model. J Oral Maxillofac Surg 2000; 58: 56-61

${ }^{49}$ Alpert B, Gutwald R, Schmelzeisen R. New innovations in craniomaxillofacial fixation: the 2.0 lock system. Keio J Med 2003; 52: $120-$ 127 
50 Egol KA, Kubiak EN, Fulkerson E et al. Biomechanics of locked plates and screws. J Orthop Trauma 2004; 18: 488-493

51 Luhr HG. Fractures of the atrophic Mandible. In: Hausmann, J-E, Ward Booth P, Schendel SA, eds. Maxillofacial Surgery. Edinburgh: Churchill Livingstone; 1999: 195-206

52 Levine PA. AO compression plating technique for treating fractures of the edentulous mandible. Otolaryngol Clin North Am 1987; 20: 457-477

53 Krebs FJ 3rd. Dynamic compression plating in treatment of the fractured, edentulous mandible. Laryngoscope 1988; 98: 198-201

54 Obwegeser HL, Sailer HF. Another way of treating fractures of the atrophic edentulous mandible. J Oral Maxillofac Surg 1973; 1: 213-221

55 Blume 0, Seper L, Meyer U et al. Experimentelle und klinische Studie zur Stabilität einer Osteosyntheseplatte für den atrophen Unterkiefer. Mund Kiefer Gesichtschir 2003; 7: 323-329

56 Seper L, Piffko J, Joos U et al. Treatment of fractures of the atrophic mandible in the elderly. J Am Geriatr Soc 2004; 52: 1583-1584

57 Joos U, Piffko J, Meyer U. Neue Aspekte in der Versorgung von Unterkieferfrakturen. Mund Kiefer Gesichtschir 2001; 5: 2-16

58 Militsakh ON, Wallace DI, Kriet ID et al. Use of the $2.0 \mathrm{~mm}$ locking reconstruction plate in primary oromandibular reconstruction after composite resection. Otolaryngol Head Neck Surg 2004; 131: 660-665

59 Farwell DG, Kezirian EJ, Heydt JL et al. Efficacy of small reconstruction plates in vascularized bone graft mandibular reconstruction. Head Neck 2006; 28: 573-579

60 Ellis E 3rd, Graham J. Use of a $2.0 \mathrm{~mm}$ locking plate/screw system for mandibular fracture surgery. J Oral Maxillofac Surg 2002; 60: 642-645

61 Novelli G, Sconza C, Ardito E et al. Surgical treatment of the atrophic mandibular fractures by locked plates systems: our experience and a literature review. Craniomaxillofac Trauma Reconstr 2012; 5: 65-74

62 Van Sickels JE, Cunningham LL. Management of atrophic mandible fractures: are bone grafts necessary? Journal Oral Maxillofac Surg 2010; 68: 1392-1395

63 Madsen MJ, Kushner GM, Alpert B. Failed fixation in atrophic mandibular fractures: The case against miniplates. Craniomaxillofacial Trauma and Reconstruction 2011; 4: 145150

64 Aziz SR, Najjar T. Management of the edentulous/atrophic mandibular fracture. Atlas Oral Maxillofac Surg Clin North Am 2009; 17: 7579

65 Spiessl B. Nonunion problems in fractures of the atrophied mandible. SSO Schweiz Monatsschr Zahnheilkd 1980; 90: 627-632

${ }^{66}$ Buchbinder D. Treatment of fractures of the edentulous mandible, 1943 to 1993: a review of the literature. J Oral Maxillofac Surg 1993; 51: 1174-1180

67 Wittwer G, Adeyemo WL, Turhani D et al. Treatment of atrophic mandibular fractures based on the degree of atrophy-experience with different plating systems: a retrospective study. J Oral Maxillofac Surg 2006; 64: 230-234

68 Santos GS, de Assis Costa MD, de Oliveira Costa $C$ et al. Failure of miniplate osteosynthesis for the management of atrophic mandibular/ fracture. J Craniofac Surg 2013; 24: e415e418

69 Vajgel A, Camargo IB, Willmersdorf RB et al. Comparative finite element analysis of the biomechanical stability of 2.0/fixation plates in atrophic mandibular fractures. J Oral Maxillofac Surg 2013; 71: 335-342

70 Madsen MJ, Haug RH. A biomechanical comparison of 2 techniques for reconstructing atrophic edentulous mandible fractures. J Oral Maxillofac Surg 2006; 64: 457-465

71 Madsen MJ, McDaniel CA, Haug RH. A biomechanical evaluation of plating techniques used for reconstructing mandibular symphysis/parasymphysis fractures. J Oral Maxillofac Surg 2008; 66: 2012-2019

72 Müller S, Burgers R, Ehrenfeld M et al. Macroplate fixation of fractures of the edentulous atrophic mandible: immediate function and masticatory rehabilitation. Clinical oral investigations 2011; 15: 151-156
Prof. Dr. med. Dr. med. dent. Carl-Peter Cornelius

Oberarzt der Klinik

Dr. med. dent. Florian Andreas Probst

Assistenzarzt der Klinik

Dr. med. dent. Jean Paul Christian

Rathgeber

Zahnarzt

Klinik und Poliklinik für Mund-,

Kiefer- und Gesichtschirurgie

Klinikum der Universität München

Ludwig-Maximilians-Universität München

Lindwurmstraße 2 a

80337 München

Priv.-Doz. Dr. med. Dr. med. dent. Marc Metzger

Oberarzt

Klinik und Poliklinik für Mund-, Kiefer- und Gesichtschirurgie Universitätsklinikum Freiburg Albert-Ludwigs-Universität Freiburg Hugstetterstraße 55

79106 Freiburg

peter.cornelius@

med.uni-muenchen.de 